\title{
Developmental Outcomes of Children Born After Assisted Reproductive Technologies
}

\author{
Jane Squires, PhD; Paul Kaplan, MD
}

\begin{abstract}
Since the birth of Louise Brown in England more than 25 years ago, an estimated 2 million children have been born worldwide through in vitro fertilization and related assisted reproduction technology (ART) procedures. Most children born after ART are healthy and develop without complications. Largely because of multiple births (twins, triplets, etc), however, some ART offspring experience developmental problems. In addition, recent studies suggest increased risk of prematurity and smaller birth weight after ART. Regular periodic screening and careful developmental follow-up of ART offspring are recommended as well as rigorous follow-up studies of children conceived after these procedures. Key words: assisted reproductive technologies, developmental outcomes, intracytoplasmic sperm injection, in vitro fertilization
\end{abstract}

$\mathbf{T}$ HE birth of Louise Brown in 1978 (Edwards, Steptoe, \& Purdy, 1980) ushered in a new era for couples having difficulty conceiving a child. Long used in the animal world, assisted reproductive technology (ART) allows scientists to manipulate the fertilization process in order to bypass barriers in women (eg, blocked fallopian tubes, damaged ovaries) and men (eg, low sperm count) to achieve pregnancy. Each year thousands of couples conceive children through procedures that would have been impossible even a decade ago. Worldwide, more than 2 million children have been born through in vitro fertilization (IVF) and related ART procedures.

Most ART offspring are healthy, happy, typically developing children, with cognitive and behavioral outcomes similar to their naturally conceived peers. Largely because of multiple births (twins, triplets, etc), how-

From the Early Intervention Program, University of Oregon, Eugene, Ore (Dr Squires); and the University of Oregon Health Center, Eugene, Ore, and the Oregon Health and Sciences University, Portland, Ore (Dr Kaplan).

Corresponding author: Jane Squires, PbD, Early Intervention Program, University of Oregon, Eugene, OR 97403 (e-mail: jsquires@uoregon.edu). ever, some ART offspring experience developmental problems. With increased numbers of multiple births and more intense human intervention and manipulation, new emphasis has been placed on developmental followup of children born after IVF and related procedures.

Research studies on the developmental outcomes of children born after ART are summarized in this review. First, current ARTs are defined, and second, potential developmental problems resulting from ART described. Third, results of follow-up studies on children born after ART are summarized. Finally, conclusions and recommendations for further research are presented. For the purposes of this review, ART will be limited to conventional IVF and IVF with intracytoplasmic sperm injection (IVF/ICSI) and related processes.

\section{WHAT ARE ASSISTED REPRODUCTIVE TECHNOLOGIES?}

\section{In vitro fertilization}

$A R T$ refers to the advanced techniques used to bring eggs and sperm together in order to help infertile couples achieve pregnancy. The most frequently used ART technique is IVF, sometimes called the "test tube baby 
Table 1. Description of assisted reproductive technologies (ARTs)*

\begin{tabular}{|ll|}
\hline ART procedure & \multicolumn{1}{c|}{ Description } \\
\hline IVF & $\begin{array}{c}\text { Oocytes (eggs) retrieved from woman's ovaries via ultrasound-guided needle } \\
\text { aspiration and mixed with husband's (or donor's) sperm in laboratory; } 1 \text { to } 4 \\
\text { embryos transferred back into woman's uterus } 3 \text { to } 6 \text { days later } \\
\text { IVF/ICSI }\end{array}$ \\
Variation of IVF in which each egg is fertilized by direct injection of a single \\
sperm \\
Eggs and sperm (gametes) are isolated and transferred directly into the fallopian \\
ZIFT & $\begin{array}{l}\text { Fubes by laparoscopy, where conception takes place } \\
\text { laparoscopy and GIFT in which embryos are transferred into fallopian tubes by } \\
\text { Excess embryos frozen after IVF and IVF/ISCI are later thawed and transferred } \\
\text { Fack into woman's uterus }\end{array}$ \\
\end{tabular}

*IVF indicates in vitro fertilization; IVF/ICSI, in vitro fertilization with intracytoplasmic sperm injection; GIFT, gamete intrafallopian transfer; ZIFT, zygote intrafallopian transfer; and FET, frozen embryo transfer.

procedure." IVF gets its name from the fact that fertilization occurs outside of the body, in vitro-in a laboratory dish. Couples turn to IVF when problems such as damaged fallopian tubes, endometriosis, or abnormal semen parameters (eg, low sperm counts) prevent pregnancy from taking place. In addition, IVF is used for unexplained infertility and failure to respond to other treatments.

IVF begins with intensive fertility drug therapy for the woman in order to stimulate the simultaneous development of multiple oocytes (eggs). The oocytes are then retrieved from the ovaries with a needle guided by transvaginal ultrasound and are mixed with the husband's (or donor's) sperm. After being grown for several days in a laboratory, developing embryos are transferred back into the uterus of the woman with a small plastic catheter. Extra embryos are cryopreserved in the laboratory for later use in frozen embryo transfer cycles.

Variations in IVF include gamete intrafallopian transfer, in which the gametes (eggs and sperm) are isolated and transferred by laparoscopy directly into the fallopian tubes where conception takes place spontaneously, and zygote intrafallopian transfer, a fusion of IVF and gamete intrafallopian transfer, in which embryos are transferred into the fal- lopian tubes. Women in the United States undergo approximately 100,000 cycles of IVF annually, with live birth rates from 35\% to $50 \%$ per cycle (Wright, Chang, Jeng, \& Macaluso, 2006). Descriptions of IVF and related procedures are summarized in Table 1.

\section{IVF with intracytoplasmic sperm injection}

With conventional IVF, eggs and sperm are mixed in a Petri dish in the laboratory and allowed to achieve fertilization without assistance. ICSI is a treatment variation within IVF in which each egg is fertilized directly by injecting a single sperm using a microscopic needle, as shown in Figure 1. It is a fairly new procedure, first described in 1992 by Palermo and associates in Belgium (Palermo, Joris, Devroey, \& Van Steirteghem, 1992). After fertilization and culture of the embryos for 3 to 6 days, they are transferred back into the uterus in a process similar to that described for IVF. Excess ICSI embryos, as with other excess IVF embryos, are often cryopreserved for later transfer. ICSI represents a major advancement in the treatment of male infertility, and allows infertility treatment for couples for whom conventional IVF is not an option. For example, men with no sperm (ie, azoospermia) or very low sperm counts can now 


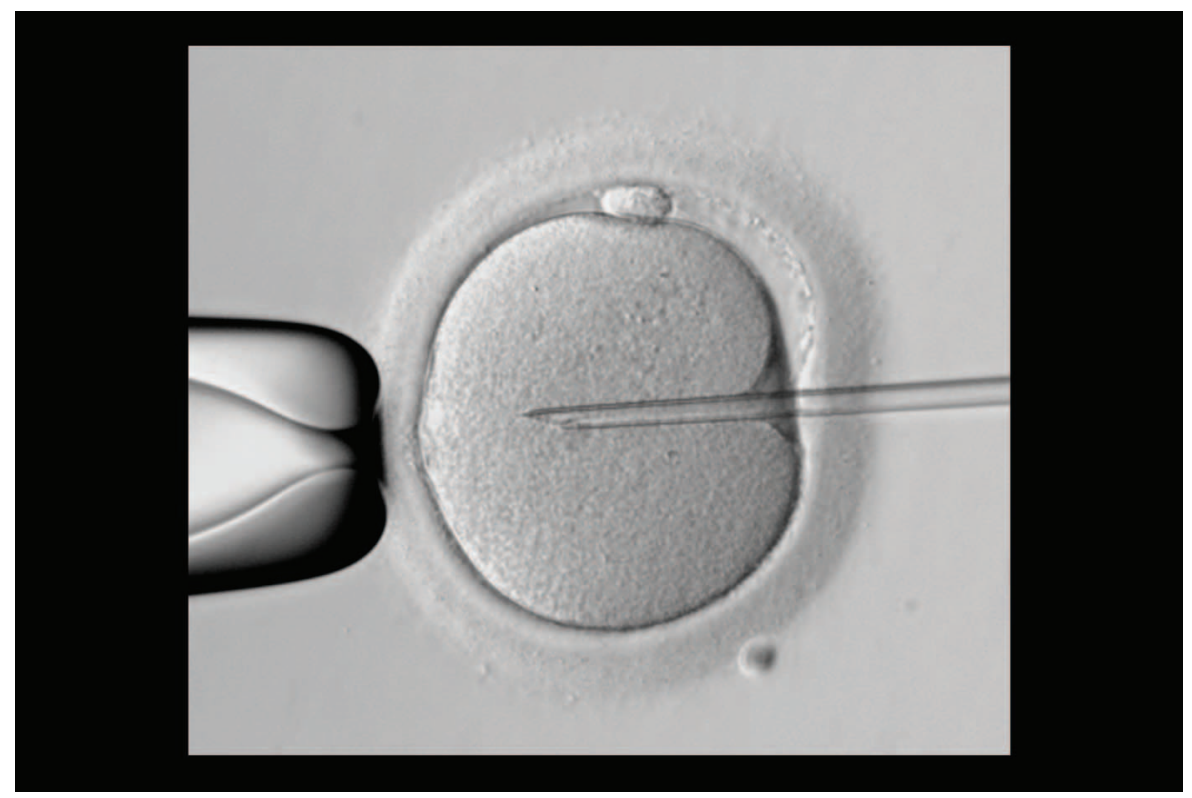

Figure 1. Intracytoplasmic sperm injection as performed in the in vitro fertilization laboratory. Courtesy of Gianpiero Palermo, MD.

conceive a child with a partner when previously donor sperm would have been required for conception.

Because each egg is fertilized by injection with a single sperm, the natural selection process that occurs during conception is bypassed with ICSI. As a result, the ICSI procedure raises 3 particular concerns for potential offspring. First, each ICSI sperm is selectively chosen by an embryologist in the laboratory and may not be a normal gamete that would participate in the natural selection process. Second, the ICSI procedure involves physical manipulation of the egg cell membrane and cytoplasm by a needle, possibly damaging the oocyte and developing embryo and increasing the risk of abnormally developing offspring (Buckett \& Tan, 2005). Third, infertile males who are candidates for ICSI have a higher incidence of Y-chromosomal abnormalities that may be passed to their offspring (Bonduelle et al., 2002). These concerns provide a strong rationale for monitoring the development of children conceived after ICSI. Approximately $47 \%$ of all IVF procedures in the United States-nearly one-half-now in- volve ICSI for egg fertilization (American Society for Reproductive Medicine/Society for Assisted Reproductive Technology Registry [ASRM/SART], 2004a).

\section{WHAT ARE THE POTENTIAL DEVELOPMENTAL PROBLEMS OF ART OFFSPRING?}

Human manipulations of egg and sperm, fertility drugs, and effects of embryo culture and transfer have the potential to affect the growing fetus. Although most studies of ART offspring to date have demonstrated no added risk for developmental problems, potential difficulties for children born after ART include (1) genetic disorders, (2) congenital anomalies, (3) preterm delivery and perinatal health issues, (4) developmental delays and disabilities, and (5) behavioral and mental health difficulties. By far, prematurity and low birth weight are the primary risk factors contributing to poor outcomes in ART offspring. Currently, 51\% of ART newborns in the United States are the result of multiple births (twins, 
triplets, and higher order births), increasing the risk of early health problems as well as long-term disability (Wright et al., 2006).

Many factors compound outcome studies investigating ART offspring. First, couples who require ART often are older-mean age of 33 years for women undergoing ART versus 27 years for women who conceive naturallyincreasing the chances of genetic problems in offspring as well as pregnancy complications (American College of Obstetrics and Gynecology, 2004). Second, most couples turn to IVF because of infertility, which may in itself increase chances of developmental problems in children because of preexisting medical and genetic problems in the parent(s). Third, mothers who undergo IVF take a combination of fertility drugs before and immediately after the procedures, which may have effects on growing embryos. Fourth, the human manipulation of sperm and egg, in vitro culture, cryopreservation, and embryo transfer may affect developmental outcomes by modifying natural processes. Finally, more than $50 \%$ of IVF births are from multiple gestations, resulting in a large negative impact on developmental outcomes (Wenstrom, Elliot, Newman, Peaceman, \& Chahaun, 2004). Outcomes associated with multiple births often need to be sorted out from general outcomes describing ART offspring. As with any research database, high-quality studies with randomized controlled designs, careful implementation, and high-quality data analyses are necessary to ensure valid and reliable results. When describing developmental outcomes, IVF will refer to conventional IVF, with unassisted fertilization of egg and sperm; IVF with ICSI for egg fertilization will be included under IVF/ICSI outcomes.

\section{WHAT DO STUDIES TELL US ABOUT EARLY DEVELOPMENT OF ART OFFSPRING?}

\section{Multiple births}

Twins, triplets, and higher order birthsmultiple fetuses sharing a uterus-greatly in- crease the rates of prematurity and low birth weight in newborns (Wenstrom et al., 2004). An estimated 49,000 infants in the United States are born annually from ART treatments, and $51 \%$ of these are from multiple births (Wright et al., 2006). Between 1980 and 1997, pregnancies in the United States resulting in twin births increased by $52 \%$, triplets and higher order births by $404 \%$, and the incidence of preterm births by $14 \%$ (Browne, 2003). Problems in offspring increase as gestational age decreases-smaller babies are usually sicker and can have more problems at birth and afterwards including cerebral palsy, learning disabilities, and visual difficulties (Hack et al., 2002; McCormick, et al., 2006). Neurodevelopmental and socioemotional deficits may persist into adolescence and young adulthood (Hack et al., 2002). Parents may also experience challenges in caring for multiple birth premature infants that can affect their developmental outcomes (Olivennes, Golombok, Ramogida, Rust, \& the Follow-up Team, 2005). Even if twins and triplets are born full term and healthy, the demands of simultaneously caring for multiple offspring may require additional supports and resources for the families. Early intervention professionals offer parenting groups and quality childcare programs that may offer needed supports to these families.

Because of dramatic increases in multiple births after IVF and associated problems in offspring, professional guidelines have strongly recommended limiting the number of transferred embryos to 1 or 2 in women younger than 35 years (ASRM/SART, 2004b), as both ongoing pregnancy rate and implantation rate per embryo are high when the oocyte donor (patient or egg donor) is in this younger age group. In some European countries, Australia, and New Zealand, legislation has been passed to limit transfer to no more than 2 embryos. In the United States, however, there are no legal restrictions, and patients often ask for the maximum number (usually 3-4) to be implanted to maximize their chance of pregnancy with these expensive procedures. 


\section{Low birth weights, increased prematurity}

Even if developmental outcomes from multiple births are excluded, children born after IVF and IVF/ICSI are more likely to be born prematurely and of lower birth weight than naturally conceived children (Geyter, De Geyter, Steimann, Zhang, \& Holzgreve, 2006). Singleton infants born after ART are twice as likely to be born preterm and 3 times more likely to weigh less than $1500 \mathrm{~g}$ (ie, slightly more than $3 \mathrm{lb}$ ) than singleton infants conceived naturally (Jackson, Gibson, Wu, \& Croughan, 2004). The reasons for the increased incidence of prematurity and low birth weight are not completely understood, but may be related to factors described above such as increased maternal age, infertilityrelated genetic anomalies, human manipulation of gametes and embryos, and use of fertility drugs. It appears that women who take longer to conceive ( $>1$ year) in general are at greater risk for having lower birth weight babies (1.5-2.0 times) than the general population, no matter the method of conception. Therefore, factors related to infertility (eg, increased age, genetic anomalies) appear to be associated with prematurity, rather than the specific ART procedures themselves (Van Voorhis, 2006).

\section{Birth defects, genetic disorders}

Although some studies have found an increased prevalence of birth defects in IVF infants, methodological errors such as failure to correct for multiple births and lack of data on confounding factors (eg, older mothers and higher education and economic attainment) have marred many studies. Several recent population-based studies with sound research designs have suggested a small increased risk for birth defects including heart malformations and limb and metabolic disorders among ART infants, including IVF as well as ICSI (eg, Klemetti et al., 2005; Olson et al., 2005). Research on birth defects can be challenging because ART children undergo much more thorough examinations as infants than is typical during well-child examinations. Often in the naturally conceived population, birth defects are identified later in childhood, at age 4 or 5 , as they become more apparent to parents and providers.

Because of increased rates of genetic disorders in men with low sperm counts who need ICSI in order to conceive, couples seeking ICSI for male infertility are often urged to consult genetic counselors before undertaking the procedure (Pauer et al., 1997). Specific sex-linked male infertility conditions passed to offspring include Klinefelter's syndrome, cystic fibrosis, and Y-chromosome deletions (eg, DAZ deletions). An increased malformation rate in the male genitalia after ICSI has also been observed in recent studies (Bonduelle et al., 2005).

Reports suggest an association between ART and congenital malformation syndromes because of genetic imprinting defects such as Beckwith-Wiedemann syndrome and Angelman syndrome (Chang, Moley, Wangler, Feinberg, \& Debaun, 2005; Sutcliffe et al., 2006). Although Beckwith-Wiedemann syndrome occurs in only $1: 12,000$ to $1: 15,000$ births, the incidence may be increased up to 4 times after ART (DeBaun, Neimitx, \& Feinberg, 2003; Maher et al., 2003). No differences in incidence of Beckwith-Wiedemann syndrome were demonstrated when comparing IVF with IVF/ICSI procedures or among different laboratory culture media conditions used in ART processes. Again, multiple confounding factors including parental age and demographics, multiple gestation, geneticassociated infertility etiologies, and small sample size in available studies preclude clear causal linkages.

\section{Developmental and behavioral outcomes}

Developmental outcomes for children are usually measured by individually administered standardized intelligence tests for children such as the Bayley Scales of Infant Development II (Bayley, 2001), Weschler Preschool and Primary Scale of Intelligence III (Wechsler, 2002), and Griffiths Mental Development Scales (Griffiths, 1984). When multiple births are excluded, some studies 
find the growth of IVF and IVF/ICSI children in terms of height and weight to be falling behind that of naturally conceived children during the first 3 years of life-probably because of their smaller size at birth. However, the motor and cognitive development of IVF and IVF/ICSI children compared with their naturally conceived peers is not significantly different (Leslie et al., 2003; Ponjaert-Kristoffersen et al., 2004). In most studies, developmental quotients or IQ scores tend to be around 100 or more for IVF, IVF/ICSI, and naturally conceived children when large groups are included and testers are blind to the status of children. As in other large outcome studies, IQ scores tend to be more related to the mother's level of education and socioeconomic status than to the mode of conception (Leslie et al., 2003).

Follow-up studies of IVF/ICSI offspring have far outnumbered those devoted to conventional IVF, because of the increased chances for anomalies due to genetic risk and human manipulation of egg and sperm. ICSI offspring have now been followed prospectively for 5 years, and their overall development has been compared with that of naturally conceived children as well as conventional IVF offspring with favorable results. In one multicountry study, 300 singleton children born after ICSI in Belgium, Sweden, and the United States were compared with matched naturally conceived children at 5 years and no significant differences were found in cognitive development or psychological well-being (Ponjaert-Kristoffersen et al., 2004).

Although initial studies suggested possible genetic as well as developmental concerns for ICSI offspring, methodological flaws limited the generalization of results. Subsequent studies have concluded that the general development of ICSI offspring is comparable to that of naturally conceived children when only singleton births are included (Papaligoura, Panopoulou-Maratou, Solman, Arvaniti, \& Sarafidou, 2004; Place \& Englert, 2003; Ponjaert-Kristoffersen et al., 2004).

Several researchers have investigated links in increased parenting stress and behavioral problems to families with IVF and ICSI offspring. Scores on the Parenting Stress Index (Abidin, 1998), Child Behavior Checklist (Achenbach, 1991), and other measures of family stress have found no significant differences in families among ICSI, IVF, and conventionally conceived children (Barnes et al., 2004; Ponjaert-Kristoffersen et al., 2004). In fact, some findings suggest that parents of IVF/ICSI offspring report less stress and fewer behavioral problems compared with parents of children conceived naturally (PonjaertKristoffersen et al., 2004; Sutcliffe, Edwards, Beeson, \& Barnes, 2004). General findings suggest few if any differences in children's behavior, parent stress, and family adjustment in families of IVF and ICSI singleton children. Table 2 summarizes developmental outcomes of IVF and ICSI children.

\section{SUMMARY AND CONCLUSIONS}

Large-scale, well-designed studies have found most children born after ART to be healthy, typically developing young children. Researchers in Europe (Katalinic, Rösch, \& Ludwig, 2004), Australia (Leslie et al., 2003), and the United States (Schieve et al., 2004; Squires, Carter, \& Kaplan, 2003) have found no significant differences between children conceived after ART and their naturally conceived peers on cognitive, motor, and behavioral assessments.

All children should receive regular periodic screening and developmental follow-up in their preschool years to ensure typical development of their cognitive, motor, language, and adaptive skills (American Academy of Pediatrics, 2006). Children conceived after ART should be included among groups that receive even more intensive developmental follow-up in their early years. Parents of ART children in the United States are often affluent, well educated, and highly motivated. Although followup studies of these children will need to correct for these demographic variables, the parents themselves will drive a demand for this more intensive developmental screening.

Periodic physician visits, as well as followalong monitoring with reliable and valid 
Table 2. Developmental outcomes of in vitro fertilization (IVF) and intracytoplasmic sperm injection (ICSI) offspring*

\begin{tabular}{|c|c|c|c|}
\hline Potential risk & $\begin{array}{c}\text { ART } \\
\text { procedure }\end{array}$ & Outcome & Supporting research \\
\hline \multirow[t]{2}{*}{ Birth defects } & IVF & $\begin{array}{l}\text { Generally no increased risk } \\
\text { reported } \\
\text { Recent studies suggest small } \\
\text { increased risk (eg, } \\
\text { cardiovascular, muscular, } \\
\text { skeletal, and genetic } \\
\text { imprinting defects) }\end{array}$ & $\begin{array}{l}\text { Place and Englert (2003); } \\
\text { Olson et al. (2005) }\end{array}$ \\
\hline & IVF/ICSI & $\begin{array}{l}\text { Recent studies suggest small } \\
\text { increased risk (eg, } \\
\text { cardiovascular, muscular, } \\
\text { skeletal, and genetic } \\
\text { imprinting defects) } \\
\text { Further research needed }\end{array}$ & $\begin{array}{l}\text { Olson et al. (2005); } \\
\text { Klemetti et al.( 2005) }\end{array}$ \\
\hline $\begin{array}{l}\text { Developmental } \\
\text { problems }\end{array}$ & $\begin{array}{l}\text { IVF and } \\
\text { IVF/ICSI }\end{array}$ & $\begin{array}{l}\text { Cognitive, motor, and verbal } \\
\text { skills similar to naturally } \\
\text { conceived peers }\end{array}$ & $\begin{array}{l}\text { Place and Englert (2003); } \\
\text { Ponjaert-Kristoffersen } \\
\text { et al. (2004) }\end{array}$ \\
\hline Behavior & $\begin{array}{l}\text { IVF and } \\
\text { IVF/ICSI }\end{array}$ & $\begin{array}{l}\text { Children's behavior, parent } \\
\text { stress, and family adjustment } \\
\text { similar to naturally conceived } \\
\text { peers }\end{array}$ & $\begin{array}{l}\text { Barnes et al. (2004); } \\
\text { Ponjaert-Kristoffersen } \\
\text { et al. (2004) }\end{array}$ \\
\hline $\begin{array}{l}\text { Prematurity and } \\
\text { low birth } \\
\text { weight }\end{array}$ & $\begin{array}{l}\text { IVF and } \\
\text { IVF/ICSI }\end{array}$ & $\begin{array}{l}\text { Increased risk for ART offspring, } \\
\text { although children's growth } \\
\text { catches up by age } 3\end{array}$ & Jackson et al. (2004) \\
\hline Multiple births & $\begin{array}{l}\text { IVF and } \\
\text { IVF/ICSI }\end{array}$ & $\begin{array}{l}\text { Nearly } 51 \% \text { of ART offspring are } \\
\text { from multiple births } \\
\text { Increased risk of prematurity, } \\
\text { medical complications, and } \\
\text { neurodevelopmental and } \\
\text { socioemotional deficits } \\
\text { By far greatest risk for ART } \\
\text { offspring }\end{array}$ & Olivennes et al. (2005) \\
\hline
\end{tabular}

*ART indicates assisted reproductive technology.

screening instruments (eg, Ages \& Stages Questionnaires: Squires, Bricker, \& Twombly, 1999; Parent Evaluation of Developmental Skills: Glascoe, 1998), are suggested so that developmental problems can be identified in a timely manner.

Any developmental delays in cognitive, motor, and socioemotional skills resulting from low birth weight or prematurity can be identified through early screening assessments, and the child and family can be referred to the needed intervention services. Identifying delays before they become major problems will improve outcomes for the child and family and save educational and psychological resources in the long term.

As ART procedures continue to evolve, with inclusion of new medications, laboratory culture conditions, and more intensive and invasive embryo diagnostic techniques, continued research on developmental outcomes of offspring is critical, especially in the United 
States where less than $5 \%$ of outcome studies have been conducted. One such example of a new technique is preimplantation genetic diagnosis (PGD), in which 1 to 2 cells are removed from the 3-day-old embryo for genetic analysis. Although it allows the opportunity to overcome chromosomal disorders through the transfer of only genetically screened embryos, the long-term safety of PGD and other new ART procedures can only be affirmed by developmental screening of the resultant children. Although not highlighted in this review, follow-up studies on offspring resulting from frozen embryo transfer, which comprise 13\% of IVF births (ASRM/SART, 2004a), egg donation IVF, oocyte (egg) cryopreservation, and in vitro maturation of oocytes are also needed to ensure the safety of these and other new procedures. IVF and related fertility pro- cedures have become remarkably successful in a short time and have risen to a position of prominence in mainstream infertility care. It is imperative that we ensure that the demand for these services and the rapid technological advances in this field do not exceed our ability to understand the potential long-term effects of these procedures on children.

The first ART offspring are only now approaching their reproductive years, and the real test may come only when ART offspring are themselves parents. If their reproductive experiences are normal and their offspring are typically developing, parents and researchers alike will be assured that this technology is safe and has realized its potential. Rigorous follow-up studies and careful developmental monitoring are critical in the developing years of this powerful technology.

\section{REFERENCES}

Abidin, R. (1998). Parenting Stress Index test manual (3rd ed.). Charlottesville, VA: Pediatric Psychology Press.

Achenbach, T. (1991). Child Behavior Checklist/2-3 years (CBCL/2-3). Burlington: Department of Psychiatry, University of Vermont.

American Academy of Pediatrics, Council on Children with Disabilities. (2006). Identifying infants and young children with developmental disorders in the medical home: An algorithm for developmental surveillance and screening. Pediatrics, 118(1), 405-420.

American College of Obstetrics and Gynecology. (2004). Prenatal diagnosis of fetal chromosomal abnormalities (Practice Bulletin Number 27, May 2001). Compendium of selected publications (pp. 605-615). Washington, DC: Author.

American Society for Reproductive Medicine/Society for Assisted Reproductive Technology Registry. (2004a). Assisted reproductive technology in the United States: 2000 results generated from the American Society for Reproductive Medicine/Society for Assisted Reproductive Technology Registry. Fertility and Sterility, 81(5), 1207-1220.

American Society for Reproductive Medicine/Society for Assisted Reproductive Technology Registry. (2004b). Guidelines on the number of embryos transferred. Fertility and Sterility, 82(3), 773-774.

Barnes, J., Sutcliffe, A., Kristoffersen, I., Loft, A., Wennerholm, U., Tarlatzis, B., et al. (2004). The influence of assisted reproduction on family functioning and children's socio-emotional development: Results from a
European study. Human Reproduction, 19(6), 14801487.

Bayley, N. (2001). The Bayley Scales of Infant Development II. San Antonio, TX: Psychological Corporation.

Bonduelle, M., Liebaers, I., Deketelaere, V., Derde, M., Camus, M, Devroey, P., et al. (2002). Neonatal data on a cohort of 2889 infants born after ICSI (1991-1999) and of 2995 infants born after IVF (1983-1999). Human Reproduction, 17, 671-694.

Bonduelle, M., Wennerholm, U., Loft, A., Tarlatzis, B., Peters, C., Henriet, S., et al. (2005). A multi-centre cohort study of the physical health of 5-year-old children conceived after intracytoplasmic sperm injection, in vitro fertilization and natural conception. Human Reproduction, 20(2), 413-419.

Browne, J. (2003). New perspectives on premature infants and their parents. Zero to Three, 24(2), 412.

Buckett, W., \& Tan, S. (2005). Congenital abnormalities in children born after assisted reproductive techniques: How much is associated with the presence of infertility and how much with its treatment? Fertility and Sterility, 84(5), 1318-1319.

Chang, A., Moley, K., Wangler, P., Feinberg, A., \& Debaun, M. (2005). Association between BeckwithWiedemann syndrome and assisted reproductive techniques: Case series of nineteen patients. Fertility and Sterility, 83(2), 349-354.

DeBaun, M., Neimitx, E., \& Feinberg, A. (2003). Association of in vitro fertilization with BeckwithWiedemann syndrome and epigenetic alterations of 
LIT1 and H19. American Journal Human Genetics, 72, 156-160.

Edwards, R., Steptoe, P., \& Purdy, J. (1980). Establishing full-term human pregnancies using cleaving embryos grown in vitro. British Journal of Obstetrics and Gynecology, 87, 737-756.

Geyter, C., De Geyter, M., Steimann, S., Zhang, H., \& Holzgreve, W. (2006). Comparative birth weights of singletons born after assisted reproduction and natural conception in previously infertile women. Human Reproduction, 21(3), 705-712.

Glascoe, F. (1998). Collaborating with parents: Using parents' evaluation of developmental status to detect and address developmental and behavioral problems. Nashville, TN: Ellsworth \& Vandermeer.

Griffiths, R. (1984). Griffiths Mental Development Scales. Bucks, UK: Association for Research in Infant and Child Development.

Hack, M., Flannery, D., Schluchter, M., Cartar, L., Borawski, E., \& Klein, N. (2002). Outcomes in young adulthood for very-low birth-weight infants. The New England Journal of Medicine, 346(3), 149-157.

Jackson, R., Gibson, K., Wu, Y., \& Croughan, M. (2004). Perinatal outcomes in singletons following in vitro fertilization: A meta-analysis. The American College of Obstetricians and Gynecologists, 103(3), 551-563.

Katalinic, A., Rösch, C., \& Ludwig, M. (2004). Pregnancy course and outcome after intracytoplasmic sperm injection: A controlled, prospective cohort study. Fertility and Sterility, 81(6), 1604-1616.

Klemetti, R., Gissler, M., Phil, D., Sevǒn, T., Koivurova, M., Ritvanen, A., et al. (2005). Children born after assisted fertilization have an increased rate of major congenital anomalies. Fertility and Sterility, 84(5), 1300-1307.

Leslie, G., Gibson, F., McMahon, C., Cohen, J., Saunders, D., \& Tennant, C. (2003). Children conceived using ICSI do not have an increased risk of delayed mental development at 5 years of age. Human Reproduction, 18(10), 2067-2072.

Maher, E., Brueton, L., Bowdin, S., Luharia, A., Cooper, W., Cole, T., et al. (2003). Beckwith-Wiedemann syndrome and assisted reproduction technology (ART). Journal of Medicine and Genetics, 40, 62-64.

McCormick, M., Brooks-Gunn, J., Buka, S., Goldman, J., Yu, J., Salganik, M., et al. (2006). Early intervention in low birth weight premature infants: Results at 18 years of age for the infant health and development program. Pediatrics, 117, 771-780.

Olivennes, F., Golombok, S., Ramogida, C., Rust, J., \& the Follow-up Team. (2005). Behavioral and cognitive development as well as family functioning of twins conceived by assisted reproduction: Findings from a large population study. Fertility and Sterility, 84(3), 725733.

Olson, C., Keppler-Noreuil, K., Romitti, P., Budelier, W., Ryan, G., \& Sparks, A. (2005). In vitro fertilization is associated with an increase in major birth defects. Fertility and Sterility, 84(5), 1308-1315.

Palermo, G., Joris, H., Devroey, P., \& Van Steirteghem, A.
(1992). Pregnancies after intracytoplasmic injection of single spermatozoon into an oocyte. The Lancet, 340 , 17-18.

Papaligoura, Z., Panopoulou-Maratou, O., Solman, M., Arvaniti, K., \& Sarafidou, J. (2004). Cognitive development of 12 month old Greek infants conceived after ICSI and the effects of the method on their parents. Human Reproduction, 19(6), 1488-1493.

Pauer, H., Hinney, B., Michelmann, H., Krasemann, E., Zoll, B, \& Engel, W. (1997). Relevance of genetic counseling in couples prior to intracytoplasmic sperm injection. Human Reproduction, 12, 1909-1912.

Place, I., \& Englert, Y. (2003). A prospective longitudinal study of the physical, psychomotor, and intellectual development of singleton children up to 5 years who were conceived by intracytoplasmic sperm injection compared with children conceived spontaneously and by in vitro fertilization. Fertility and Sterility, $80(6)$, 1388-1397.

Ponjaert-Kristoffersen, I., Tjus, T., Nekkebroeck, J., Squires, J., Verte, D., Heimann, M., et al. (2004). Psychological follow-up of 5-year-old ICSI children. $\mathrm{Hu}$ man Reproduction, 19(12), 2791-2797.

Schieve, L., Rasmussen, S., Buck, G., Schendel, D., Reynolds, M., \& Wright, V. (2004). Are children born after assisted reproductive technology at increased risk for adverse health outcomes? The American College of Obstetricians and Gynecologists, 103(6), 1154-1163.

Squires, J., Bricker, D., \& Twombly, L. (1999). Ages \& Stages Questionnaires. Baltimore: Brookes.

Squires, J., Carter, A., \& Kaplan, P. (2003). Developmental monitoring of children conceived by intracytoplasmic sperm injection and in vitro fertilization. Fertility and Sterility, 79(3), 453-454.

Sutcliffe, A., Edwards, P., Beeson, C., \& Barnes, J. (2004). Comparing parents' perceptions of IVF conceived children's behavior with naturally conceived children. Infant Mental Health Journal, 25(2), 163-170.

Sutcliffe, A., Peters, C., Bowdin, S., Temple, K., Reardon, W., Wilson, L., et al. (2006). Assisted reproductive therapies and imprinting disorders-A preliminary British survey. Human Reproduction, 21(4), 10091011.

Van Voorhis, B. J. (2006). Outcomes from assisted reproductive technology. Obstetrics \& Gynecology, 107(1), 183-200.

Wechsler, D. (2002). Weschler Preschool and Primary Scale of Intelligence III. New York: The Psychological Corporation.

Wenstrom, K., Elliot, J., Newman, R., Peaceman, A., \& Chahaun, S. (2004). Multiple gestation: Complicated twin, triplet, and high-order multifetal pregnancy. American College of Obstetricians and Gynecologists, 104(4), 869-883.

Wright, V., Chang, J., Jeng, G., \& Macaluso, M. (2006). Assisted reproductive technology surveillance-United States, 2003. MMWR Surveillance Summaries, 55(SS04), 1-22.

\section{Current articles- http://depts.washington.edu/isei/iyc/iyc_comments.html}

